\title{
Treewidth and Hyperbolicity of the Internet
}

\author{
Fabien de Montgolfier* \\ LIAFA, Université Paris Diderot \\ Email: fm@liafa.jussieu.fr
}

\author{
Mauricio Soto* \\ LIAFA, Université Paris Diderot \\ Email: mausoto@liafa.jussieu.fr
}

\author{
Laurent Viennot* \\ INRIA \\ Email: Laurent.Viennot@inria.fr
}

\begin{abstract}
We study the measurement of the Internet according to two graph parameters: treewidth and hyperbolicity. Both tell how far from a tree a graph is. They are computed from snapshots of the Internet released by CAIDA, DIMES, AQUALAB, UCLA, Rocketfuel and Strasbourg University, at the AS or at the router level. On the one hand, the treewidth of the Internet appears to be quite large and being far from a tree with that respect, reflecting some high degree of connectivity. This proves the existence of a well linked core in the Internet. On the other hand, the hyperbolicity (as a graph parameter) appears to be very low, reflecting a tree-like structure with respect to distances. Additionally, we compute the treewidth and hyperbolicity obtained for classical Internet models and compare with the snapshots.
\end{abstract}

\section{INTRODUCTION}

\section{A. Motivation}

Understanding the structure of the Internet covers several aspects. One is to understand the building process of the Internet, another is to design faithful models for simulation, a third one is to measure its properties to better tune its protocols. We are mostly concerned by this last point. Our approach was guided by finding properties that make Internet tractable, but we are also interested in properties measuring how robust is the topology. Indeed, many graph problems have better algorithmic solutions when the input is a graph with some known properties rather than an arbitrary graph. Better knowing the Internet thus enables better algorithms. We focus on two graph parameters known to bring tractability (in the sense that hard problems can be quickly solved if the parameter is low): treewidth and hyperbolicity. These parameters measure how far is a graph from a tree from the point of view of connectivity and distances, respectively.

The treewidth parameter is interesting for two reasons. First a low treewidth is known to enable linear-time algorithms for many problems that are NP-hard in the general case [1]. Second it is a measure of connectivity. The treewidth of a graph is related to the number of nodes required to significantly reduce the connectivity of the graph as described later on. For example, a tree (which always has treewidth equal to one) can be disconnected by removing a single node. A higher treewidth is the sign of a better connectivity.

On the other hand, the hyperbolicity parameter is related to distances: a graph is close to a tree if routes between vertices behave like in a tree. The interest of measuring hyperbolicity

*INRIA Project-Team "GANG" joint with Université Paris Diderot and CNRS. Supported by "EULER" european project (Grant No.258307). is twofold. First, similar properties have already been observed on the Internet [2]-[4]. Second, graphs with low hyperbolicity offer more tractability, enabling efficient algorithms for routing related problems like compact routing, or diameter estimation, among others [5]-[8].

The tractability issues of Internet growth mainly concern the AS (Autonomous Systems) level routing. The global connectivity of the Internet is also mainly concerned with this graph: for instance, how many ASes a cyber-attacker has to infect in order to significantly disconnect the Internet?

The Internet can be seen either as a network of networks (the AS-level Internet) or as a collection of networks. This fact results in three types of graphs that are interesting with respect to our parameter measures: the AS level graph, where each AS is viewed like a node and two different Ases are connected if they interchange data traffic; the router level graph, modeling the connections of all Internet routers; and finally the induced graph within an individual AS. These structures are not publicly available and represent and important research field. It is well known that it is difficult (if ever possible) to obtain an accurate view of these Internet graphs. We use different existing sources of data obtained using varied techniques. Nevertheless, most strategies are mainly based in traceroute probing or in the analyse of BGP (Border Gateway Protocol) routing tables.

These kinds of snapshots are known to be incomplete and lack accuracy [9], [10]. However, this is the best data available as far as we know. On the other side, our measures have some robustness properties with respect to missing nodes and edges. More precisely, the treewidth of a graph is at least the treewidth of any of its subgraphs (see third section). The data comes from six different sources (detailed in Section II), namely: CAIDA [11], DIMES [12], AQUALAB [13], UCLA (Internet Topology Collection) [10], Rocketfuel project [14] and Strasbourg University (MRINFO Project) [15].

\section{B. Contribution}

Our first conclusion is that the treewidth of the Internet is high: all the snapshots have a treewidth comparable to that of a square grid with same number of nodes (a grid is a classical example of sparse graph with high treewidth). From that point of view, Internet is far from a tree: it is much better connected. This result gives an estimation of the connectivity of the Internet: there exists a core that cannot be broken in two parts by removing less than $t w / 2$ ASes where $t w$ is the treewidth. This result holds independently of the accuracy of 
the measure of the graph: discovering more nodes or links can only increase $t w$.

Our second conclusion is that the hyperbolicity of the Internet is low, confirming previous results [2]-[4]. Conversely to this previous work, the hyperbolicity definition we use has algorithmic applications [5]-[7] such as enabling compact routing with small additive stretch [8]. From that point of view, Internet is close to a tree and far from a grid.

Additionally, we observe an important churn at the AS level. This can explain why the set of observed IP addresses increases with time in previous work [16].

Finally, we compare these measurements with classical Internet models based on various type of random graph generation. These graphs present a slightly higher treewidth and similar hyperbolicity. The closest models are those obtained from random generation with appropriate degree distribution. Surprisingly, the structure of Internet seems to be fairly represented by such a model. This fact was already observed [17]. The fit is particularly true for hyperbolicity. This somehow contradicts a possible interpretation of previous work [2][4] stating that low hyperbolicity is an intrinsic property of the Internet. It happens to be a usual property among graphs with similar degree distribution. On the other hand, random graphs appear to have slightly higher treewidth and thus better connectivity than the Internet. Yet the connectivity of the Internet is not so far from that of a random graph.

\section{Related Work}

Internet Topology was studied by Pansiot and Grad [18] at the router level and Govindan and Reddy [19] at the AS level. Numerous work has focused on the degree distribution observed at both levels. They are heavy tailed and can be modeled with power laws [20] or Weibull distributions [14], [21], [22].

Broido and claffy study the connectivity of Internet [21]. More specifically, they inspect how the giant strongly connected component behaves with regard to node removal. The treewidth is a theoretical parameter for measuring such a global connectivity.

The treewidth parameter was introduced by Robertson and Seymour. [23]. It is related to tractability: many NP-complete problems can be solved in linear time via dynamic programming for any class of graphs with bounded treewidth [1].

A large literature concerns the understanding of Internet delay space. Embedding Internet delay space in an Euclidean space allows to build virtual coordinate systems [24], [25]. It has been noted that embedding in an hyperbolic space instead could give better results [2]. Hyperbolicity can generally be measured on a metric or on a graph according to the definition of Gromov [26]. Ramasubramanian et al. show that a relaxed version of this definition called the four point condition matches Internet delays [3]. This property can be used for predicting Internet delays through an embedding in a tree. Other notions of dimension have been tested on Internet delays such as fractal dimension [27] or doubling dimension [28].
Gromov's notion of hyperbolicity is defined as a four point condition and applies to graphs as well. Up to our knowledge, this notion of hyperbolicity has not yet been measured on the Internet. Narayan and Saniee study an alternative definition relying on $\delta$-thin triangles [4] and measure it on Rocketfuel data [14]. We obtain similar results based on Gromov's original definition.

The discovery of heavy tail in the degree distribution has raised the question of designing adequate models for the Internet. A first goal for modeling is to understand the emergence of such heavy tail distributions. Preferential attachment was proposed by Barabási and R. Albert [29] for explaining the web graph structure, or social networks. Concerning the Internet, a possible explanation concerns the optimization of tradeoffs [30]. Another reason for modeling is to generate large network with similar properties as the Internet for simulation purposes. In that trend, generating a random graph with an appropriate degree distribution surprisingly appears to fit well with regard to structural properties [17]. Our work gives also credit to that point.

\section{Roadmap}

Section II describes the snapshots of the Internet we have used for our measurements and makes a comparison of the AS snapshots over time. Section III gives the treewidth definition and its relationship with connectivity. Upper and lower bounds of the treewidth of the snapshots are given. Section IV introduces the hyperbolicity definition and describes the behavior of the snapshots. Section V compares these results to what we obtain for classical graph models.

\section{Data Sets}

Real Internet topology is unknown. Different techniques have been developed in order to obtain realistic snapshots. In the aim of bypassing the several deficiencies of data collection, we use heterogeneous data sources with diverse inference techniques. For each data source we use snapshots collected at different times (and unless otherwise specified during one month) to capture the graph dynamic.

There exist different levels of granularity for Internet. First, the router level corresponds to IP interconnection between routers. Second, the AS level corresponds to the interconnections between ASes (such links can be observed at the BGP routing level or at IP level). Finally, we look at the inner topology (at router level) of some ASes.

Data sets combine passive and active measurement techniques. BGP data collected passively (by dumping BGP routing tables at some routers) or actively through looking glasses is a main source of inter AS connectivity. For router level, traceroute is the widely used tool to discover router interconnections. Traceroute discover IP paths followed by probe packets sent from monitor routers to a list of destinations. Additionally, the IP interconnections between two IP addresses can be used to infer an AS interconnection between the ASes who advertise IP prefixes. Internet snapshots used in this work come from the sources of data described below. 


\begin{tabular}{|c|c|c|c|c|c|c|c|c|}
\hline & & & \multicolumn{3}{|c|}{ Graphs parameters } & \multicolumn{3}{|c|}{ Largest bi-connected component } \\
\hline & & & $|V|$ & Avg. Degree & $\beta$ & $|V|$ & Avg. Degree & $\beta$ \\
\hline \multirow{4}{*}{ AS graphs } & AQUALAB & $12 / 2007-09 / 2008$ & 31847 & 9.00 & 2.18 & 25341 & 10.80 & 2.18 \\
\hline & CAIDA & $12 / 2010$ & 29797 & 5.31 & 2.16 & 17559 & 7.49 & 2.16 \\
\hline & DIMES & $12 / 2010$ & 29542 & 6.84 & 2.12 & 21296 & 8.72 & 2.12 \\
\hline & UCLA & $12 / 2010$ & 37450 & 6.65 & 2.14 & 25271 & 8.73 & 2.14 \\
\hline \multirow{3}{*}{ router graphs } & CAIDA router & $04 / 2003$ & 192,244 & 6.36 & $(2.93)$ & 132,367 & 8.17 & $(2.96)$ \\
\hline & CAIDA router & $07 / 2010$ & $3,360,982$ & 2.93 & 2.27 & $1,644,761$ & 3.84 & 2.18 \\
\hline & mrinfo & 09/2008 & 8,636 & 2.72 & 3.26 & 1705 & 3.60 & 3.71 \\
\hline \multirow{9}{*}{ routers within AS \# } & \multicolumn{2}{|c|}{1221 Telstra (Australia) } & 2669 & 2.38 & 2.45 & 246 & 6.07 & 2.46 \\
\hline & \multicolumn{2}{|c|}{1239 Sprintlink (US) } & 7337 & 2.70 & 2.37 & 1054 & 6.70 & 2.77 \\
\hline & \multirow{2}{*}{\multicolumn{2}{|c|}{1755 Ebone (Europe) }} & 295 & 3.68 & 2.86 & 178 & 4.76 & 3.24 \\
\hline & & & 4670 & 3.26 & 2.59 & 1644 & 5.54 & 2.76 \\
\hline & \multicolumn{2}{|c|}{$\begin{array}{l}2914 \text { Verio (US) } \\
3257 \text { Tiscali (Europe) }\end{array}$} & 411 & 3.18 & 2.77 & 166 & 4.81 & 2.97 \\
\hline & \multicolumn{2}{|c|}{3356 Level3 (US) } & 1620 & 8.32 & 2.39 & 729 & 16.03 & 2.43 \\
\hline & \multicolumn{2}{|c|}{3967 Exodus (US) } & 375 & 4.53 & 2.85 & 254 & 5.33 & 3.22 \\
\hline & \multirow{2}{*}{\multicolumn{2}{|c|}{$\begin{array}{l}4755 \text { VSNL (India) } \\
7018 \text { AT\&T (US) }\end{array}$}} & 41 & 3.32 & 2.29 & 22 & 3.91 & 2.33 \\
\hline & & & 9430 & 2.48 & 2,65 & 1199 & 5.54 & 2.89 \\
\hline
\end{tabular}

TABLE I: Basic statistics of snapshots.

Table I shows, for each graph, its size, average degree, the size of largest bi-connected component and the exponent $\beta$ of a power law fitting the degree distribution. This exponent is obtained via a linear regression on the complementary cumulative distribution function of degree distribution.

\section{A. AS-level graphs}

Vertex-set is the AS-set and edges are inter-AS links. We use:

- CAIDA, the Cooperative Association for Internet Data Analysis. Its infrastructure consists in about twenty monitors that daily collect traceroute probes to destination in full routed address space subdivided into /24's. The AS level data set [11], periodically constructed from probes and IP to AS mapping, gives us snapshots.

- AQUALAB [13] uses peer-to-peer clients to collect traceroute paths which are used to infer AS interconnections. Probes were made between December 2007 and September 2008 from approximate 992,000 P2P users in 3,700 ASes.

- DIMES [12] project performs traceroutes from a volunteer community of about 1000 agents. A weekly AS snapshot is available.

- UCLA (Internet Topology Collection ${ }^{1}$ ) [31] collects inter AS links by combining different BGP sources both passive (Route Views, RIPE-RIS, Abilene, CERNET BGP View) and active (Packet Clearing House, UCR, traceroute.org, Route Server Wiki).

\section{B. Router-level graphs}

The vertex-set is a set of routers, and the edges are their known links, at the IP level. We use data from:

- Rocketfuel project [14], using traceroute tool. Probes are made from public servers and alias resolution is performed from BGP tables.

- MRINFO project $^{2}$ from Strasbourg University. Using mrinfo, an IGMP multicast tool, the topology is discovered by sending IGMP ASK_NEIGHBORS messages, which are

\footnotetext{
${ }^{1}$ http://irl.cs.ucla.edu/topology/

${ }^{2}$ http://svnet.u-strasbg.fr/mrinfo/index.html
}

replied with the list of interfaces of a router. This method discovers all interfaces of replying hosts and avoids alias resolution process. However, replies are obtain only in routers with IPv4 multicast enabled which reduce the set of probed nodes. For details see [15].

- CAIDA also provides router-level graphs from the Internet topology data $\mathrm{kit}^{3}$. Two snapshots are analyzed here, made in April 2003 and July 2010, respectively.

\section{Comparison of AS level snapshots}

As illustrated by Figure 1(a), the size of the AS level graph slowly increases over time. Each point corresponds to the data collected during one month. The DIMES curve seems more erratic, this may come from changes in the monitor set used.

To see the influence of the aggregation window, we plot in Figure 1(b) the number of ASes $(|V|)$ and links $(|E|)$ collected between January 1st 2010 and the end of each month of that year. The size of the aggregated AS graph increases surprisingly, denoting a high churn of reconnection at the AS level. A similar linear increase was already noticed at the IP level [16] where new IP addresses are continuously discovered when probing the same destination set from the same monitor with traceroute-like measurements. The churn we observe here at AS level provides an explanation for this: as connections between ASes change, new routers become exposed in the IP probes. A possible explanation for this AS churn is that BGP routing policies are frequently updated (at the pace of commercial interactions).

From now on, we use snapshots aggregated over one month except for AQUALAB for which we only have a snapshot aggregated over 10 months.

\section{TREEWIDTH}

\section{A. Definition}

A tree decomposition of $G=(V, E)$ consists of a tree $T$ (on a different node set than $G$ ), and a subset $V_{t} \subseteq V$ associated with each node $t$ of $T$ (called a "bag".) The tree $T$ and the collection of bags $\left\{V_{t} \mid t \in T\right\}$ must satisfy the following three

\footnotetext{
${ }^{3}$ http://www.caida.org/data/active/internet-topology-data-kit/
} 


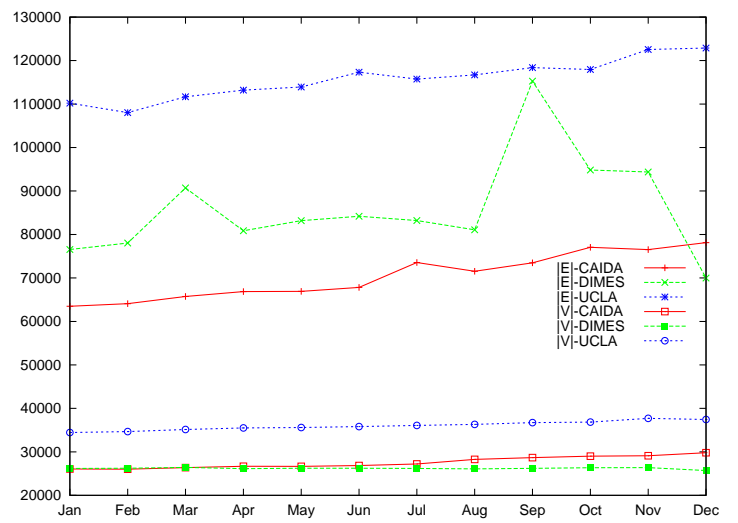

(a) Monthly snapshots.

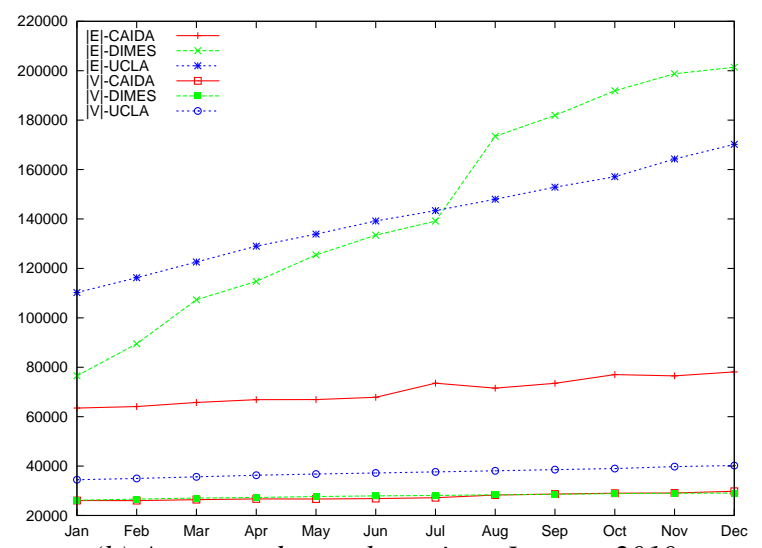

(b) Aggregated snapshots since January 2010.

Fig. 1: Size evolution of AS snapshots in 2010.

properties:

- Every node of $G$ belongs to at least one bag of $T$

- For every edge $e$ of $G$, there is some bag $V_{t}$ containing both ends of $e$.

- The collection of bags containing a given node of $G$ induces a connected subtree of $T$.

The width of the tree decomposition $\left(T,\left\{V_{t}\right\}\right)$ is defined to be one less than the maximum size of a bag: $\operatorname{width}\left(T,\left\{V_{t}\right\}\right)=$ $\max _{t}\left|V_{t}\right|-1$. The treewidth of $G$ is the minimum width of a tree decomposition of $G$ (taken over all possibles trees). A connected graph has treewidth 1 if and only if it is a tree. A tree decomposition of $G$ naturally induces a tree decomposition of any subgraph $H$ of $G$. This implies that treewidth $(H) \leq \operatorname{treewidth}(G)$. Particularly, any lower bound on the treewidth of a subgraph of $G$ is also valid for $G$. Since our graphs should be subgraphs of the "real Internet graph", we focus on computing lower bounds. In Figure 4 however, we have used other heuristics to get upper bounds so that the real value (NP-hard to compute [32]) of the snapshot lies within these bounds.

Sparse graphs ( $n$ vertices and $O(n)$ edges) may have a treewidth either low (it is 1 for a tree) or high (a square grid has treewidth $\sqrt{n}$ ).

Note that the removal of all vertices from the same internal bag of $T$ disconnects the graph $G$. Efficient algorithms use this property by removing a bag and working recursively on the remaining connected components. The treewidth can also be seen as a measure of global connectivity in the graph (see III-E).

Computing the treewidth of a graph is NP-hard [32]. However there exists heuristics for computing lower and upper bounds of the treewidth ${ }^{4}$. We have used them on our data: the results are given below. The treewidth of a graph is the maximum over biconnected components of treewidth. As our graphs have a large biconnected component and many pending trees, we work only on the giant biconnected component to increase computation speed.

\section{B. Treewidth of the AS Graphs (snapshots)}

We have computed treewidth lower bounds for the Internet snapshots we have of the inter-AS links (the $A S$ graph). Figure 2 plot the lower bound computed for each December snapshot (except in 2011 where the February snapshot is used). We have two special graphs: INTER is the links that appear in all sources and UNION the links that appear in some source (at a given date, any AS graph is thus a supergraph of INTER and a subgraph of UNION). As seen in Table I, UCLA has more ASes and so without surprise has higher treewidth. Most notably, we see that the treewidth increases over time whatever source we consider (UCLA, DIMES or CAIDA). This may be explained by the fact that the AS graph itself increases as shown by Figure 1(a).

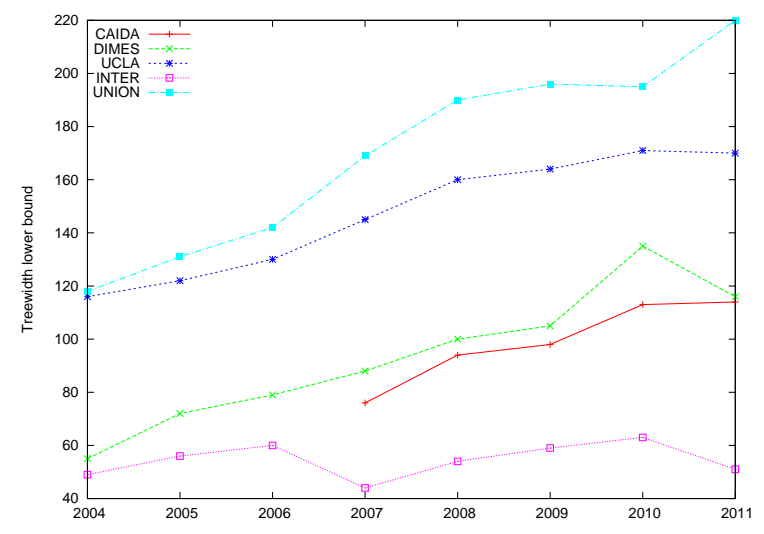

Fig. 2: Treewidth of the AS graph over time.

\section{Treewidth of the AS Graphs (long time measurement)}

AS links change over time and some data, especially from AQUALAB, are aggregated over a long period of measurement. In order to show how different the snapshots are with respect to aggregation period, we have computed, for each month of 2010, the treewidth of the graph collected between January 1st and the end of that month, and the corresponding treewidth (Figure 3, while Figure 1(b) is the size of the graph). The number of discovered edges seems to linearly increase so treewidth also increases.

\footnotetext{
${ }^{4}$ We use software from Bodlaender team at http://www.treewidth.com/
} 


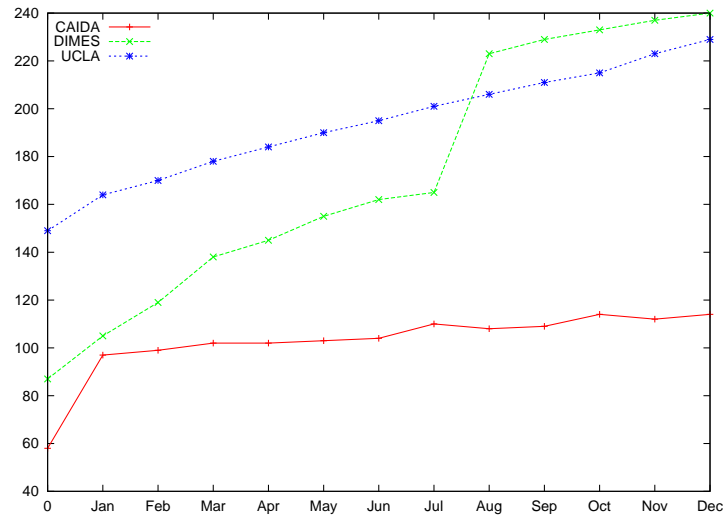

Fig. 3: Evolution of treewidth for different aggregation periods: $y=$ treewidth(links known from January 1st to Month $x)$

Table II shows the lower bound of treewidth for the various snapshots gathered. We compare it with the square root of the number of vertices, ie, the treewidth of a grid with the same number of vertices. The ratio $\operatorname{treewidth}(G) / \sqrt{n}$ appears to remain constant (close to 1 ) for each data source independently from time.

\section{Treewidth of the Router Graphs}

Figure 4 shows treewidth lower and upper bounds for router level graphs. We have computed the values for both global router-level graphs and inner ASes snapshots.

Almost all snapshots appear to have a treewidth which is close to the treewidth of a square grid with the same number of nodes. This proves a high degree of connectivity of the Internet. A noticeable exception is AS 3356 (Level3), which appears to have a very high treewidth. This probably comes from virtual circuits enabled through Multiprotocol Label Switching (MPLS), a technology in which this AS is a leader. Another exception is the MRINFO snapshot which appears to have a lower treewidth than the other snapshots. This certainly comes from the sparsity of this graph. This can be explained by the fact that in this graph are kept only nodes responding IGMP queries. It happens that few neighbors of a responding node do respond to IGMP queries.

\section{E. Conclusion: a Core of the Network}

The treewidth parameter captures how well the graph is globally connected in the following sense. It is linked to the number of nodes required to significantly reduce the connectivity of $G$. More precisely, a subset $S$ of nodes in

\begin{tabular}{|ll|c|c|}
\hline \multicolumn{2}{|c|}{ Graph } & tw & $\sqrt{|V|}$ \\
\hline AQUALAB & $12 / 2007-09 / 2008$ & 236 & 178 \\
CAIDA & $12 / 2010$ & 113 & 162 \\
DIMES & $12 / 2010$ & 135 & 156 \\
UCLA & $12 / 2010$ & 171 & 177 \\
UNION & $12 / 2010$ & 195 & 179 \\
INTERS. & $12 / 2010$ & 63 & 146 \\
\hline
\end{tabular}

TABLE II: Treewidth lower bound for AS graphs.

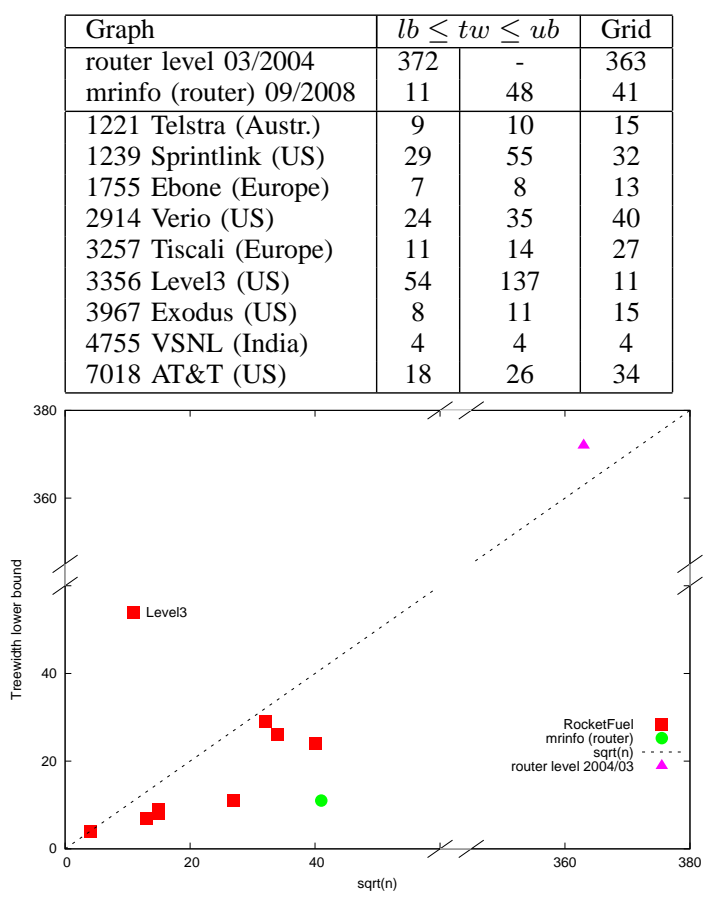

Fig. 4: Treewidth bounds for router graphs. Bottom, plot with comparison to $\sqrt{n}$

$G$ is $k$-linked if for any subset $X$ with fewer than $k$ nodes, some connected component of $G-X$ contains more than half of the nodes of $S$. In other words, the removal of less than $k$ nodes cannot drastically disconnect $S$. The linkedness of a graph is the maximum $k$ such that there exists a $k$-linked set.

The set $S$ can be seen as a core in the network which cannot be globally disconnected by the removal of fewer than $k$ nodes: more than half of the core always remains connected. The linkedness is a measure of fault tolerance of the core. Both parameters are linked according to the following theorem.

Theorem 1: [33] For any graph $G$,

linkedness $(G) \leq$ treewidth $(G)+1 \leq 2$ linkedness $(G)$.

We have chosen to estimate treewidth rather than linkedness because the lack of efficient heuristics for bounding the former. Another reason is that the lower bounds hold for the Internet as long as our snapshots remain subgraphs from it.

Lower bounds on the linkedness of our snapshots can be deduced for the table of Figure 4 (divide by 2 the lower bound of treewidth plus one). For example, the linkedness of the AS snapshot of UCLA (2011) is at least 86. This means that there exists a core of ASes that globally remains connected even under the failure of 85 ASes or less (application of Theorem 1). This gives a measure of the fault tolerance of the Internet. The core can be extracted provided a tree decomposition (given by heuristics).

\section{HYPERBOLICITY}

\section{A. Definition}

Mikhail Gromov introduced and developed hyperbolic groups in the 1980's. In a seminal paper from 1987 [26] 
he proposed a wide-ranging research program. Aiming at studying groups through their Cayley graph, he defined $\delta$ hyperbolicity. This notion may however be used apart from its group theory context.

Let $x, y, z, t$ be four vertices. Let $d_{1}, d_{2}$ and $d_{3}$ be the three sums $\operatorname{dist}(x, y)+\operatorname{dist}(z, t), \operatorname{dist}(x, z)+\operatorname{dist}(y, t)$ and $\operatorname{dist}(x, t)+\operatorname{dist}(y, z)$ non-increasingly sorted: $d_{1} \geq d_{2} \geq d_{3}$. Then define $\delta(x, y, z, t)=\frac{d_{1}-d_{2}}{2}$. The hyperbolicity (or $\delta$ hyperbolicity) of a graph $G$, denoted $\delta(G)$ or just $\delta$ when not ambiguous, is $\max _{x, y, z, t \in V(G)} \delta(x, y, z, t)$.

Like treewidth, $\delta$-hyperbolicity indeed measures how far a graph is from a tree. Trees have hyperbolicity equal to zero and conversely any graph with hyperbolicity equal to zero can be isometrically embedded into a tree. Chordal graphs, which have a tree-like structure, have hyperbolicity equal to one. However, there is no relationship between the treewidth and hyperbolicity distance-to-tree measures. For instance a complete graph has large treewidth but is 0-hyperbolic. Conversely, an $n$-cycle is $n / 4$-hyperbolic but has treewidth equal to two. A $n \times m$-grid has both treewidth and $\delta$-hyperbolicity $\min (n, m)$ and is far from a tree in both measures.

It follows from its definition that $\delta$-hyperbolicity can be computed in polynomial time. But the $O\left(n^{4}\right)$ naive implementation is slow and we use heuristics to obtain faster (and
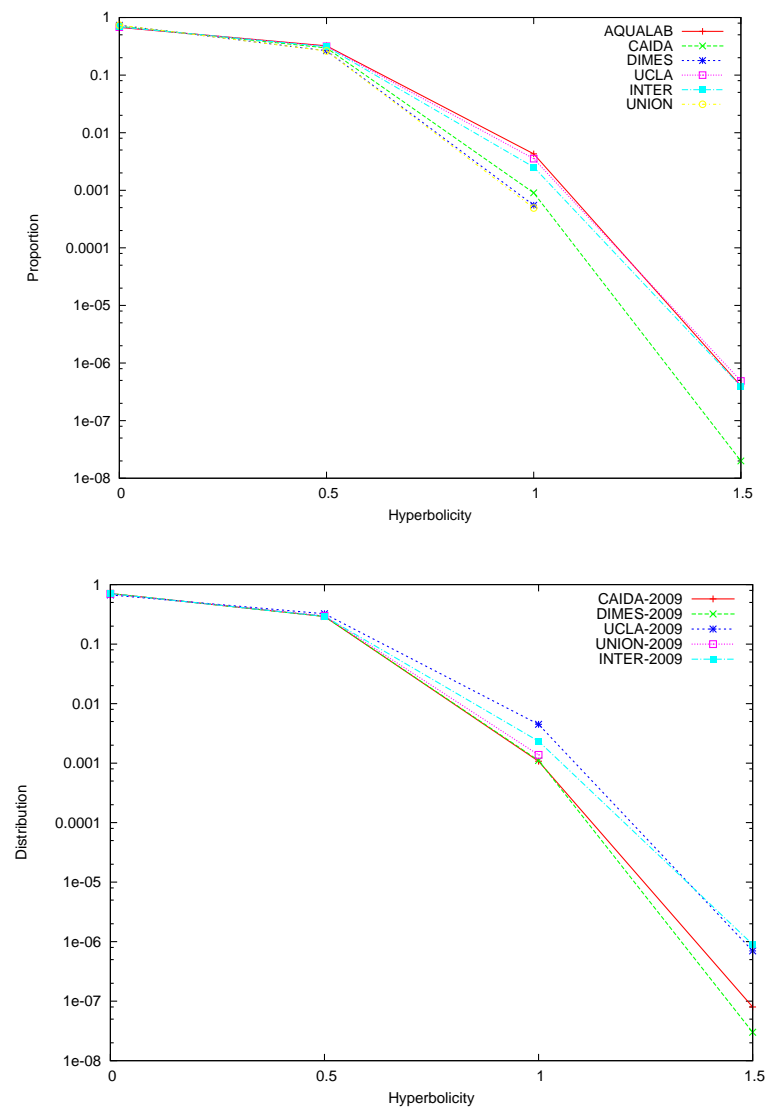

Fig. 5: Distribution of the value of the hyperbolicity $\delta(x, y, z, t)$ of quadruplets for the AS graphs (Top: 10-month aggregation. bottom: 2010/12 month snapshot) exact) computation.

Many problems can be solved efficiently for $\delta$-hyperbolic graphs (classes of bounded $\delta$-hyperbolicity). Let us cite fast diameter and center heuristics approximation (using two BFSs), and small stretch spanning tree computation [5]. Covering by balls and $k$-center, two NP-complete problems for general graphs, are addressed in [6]. A compact distance labeling (enabling to compute the distance between two nodes as a function of their labels) is given in [7]. Finally small-stretch additive spanners and compact routing computation are treated in [8].
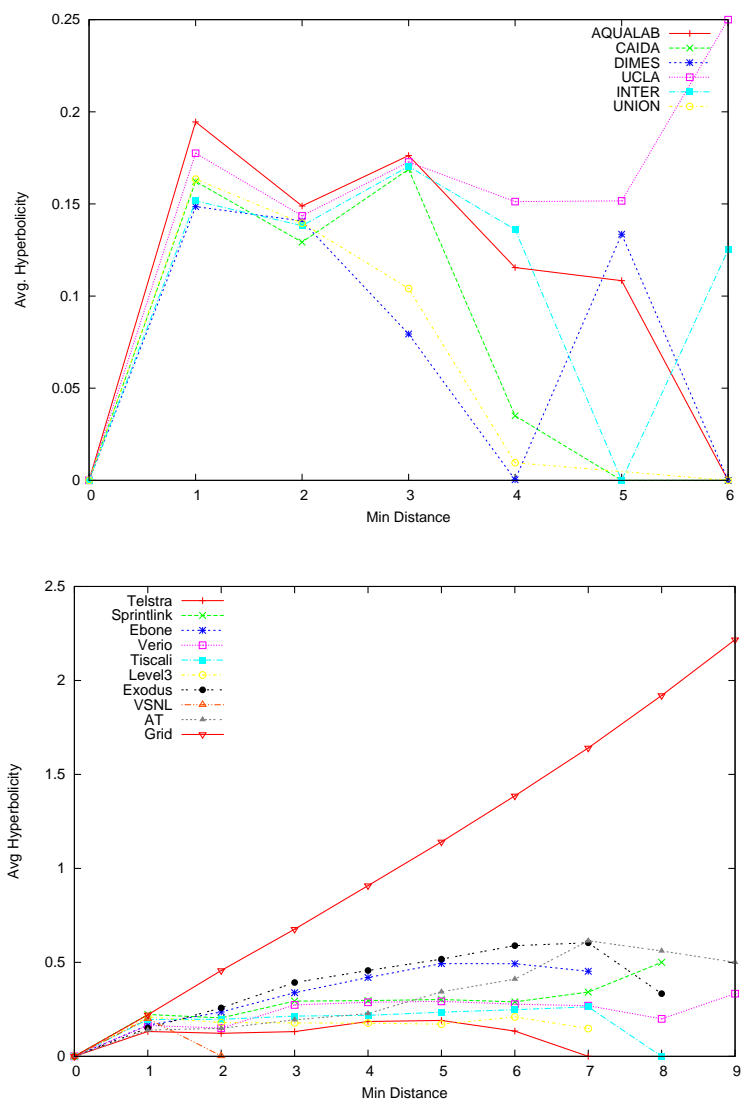

Fig. 6: Average hyperbolicity of a quadruplet with respect to the minimum distance $d_{3}\left(d_{3}\right.$ is defined in begining of currect section) Top: AS graphs. Bottom: router graphs.

\section{B. Results}

We have measured $\delta$-hyperbolicity on the same datasets than treewidth. The $\delta$-hyperbolicity of a graph is the maximum of $\delta$-hyperbolicity over its biconnected components, and since each graph contains only one non-trivial component (whose size is given in Table I), computation is performed only on this largest component to save time.

While classical definition involves only giving the maximum hyperbolicity over all quadruplets, we found that the distribution is also interesting, since it appears to exponentially decrease with $\delta$ and the maximum can be deduced easily (see Figure 5). One can observe that, for almost all 

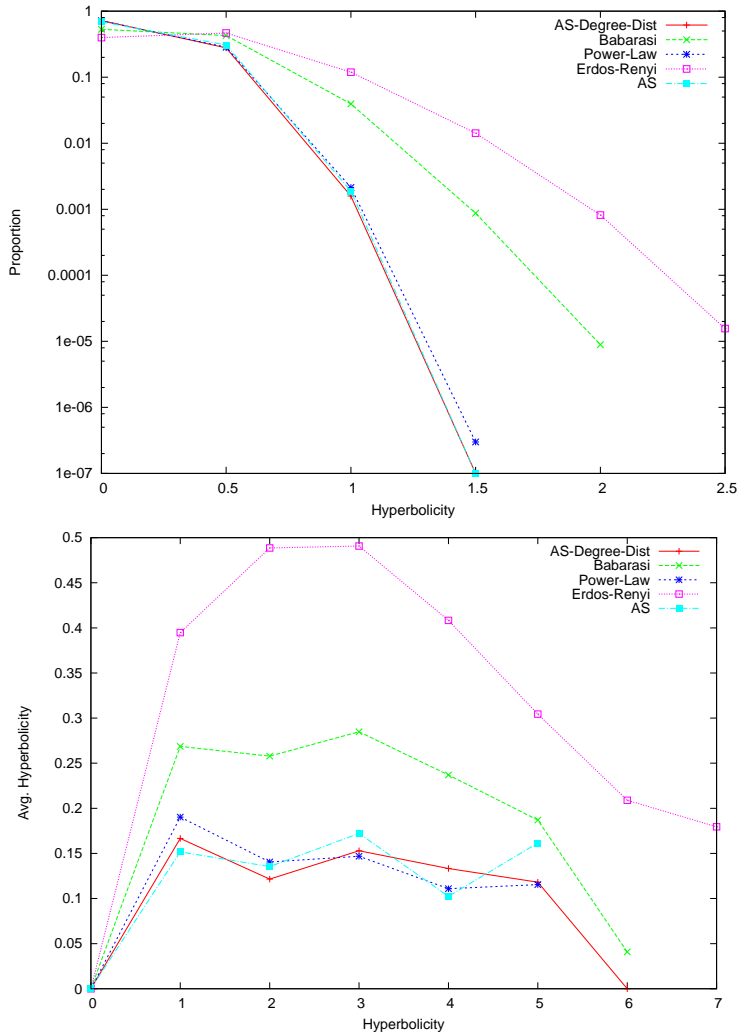

Fig. 7: Internet models. Top: percentage of quadruplets having a given hyperbolicity. Bottom: mean hyperbolicity of a quadruplet with respect to the minimum distance.

quadruplets $(x, y, z, t)$, we have $\delta(x, y, z, t) \leq 1$. Notice that if $\delta(x, y, z, t)=0$ the shortest routes between these four points can be mapped into a tree. The ASes from Rocketfuel have all a similar behavior.

The distribution of hyperbolicity appears to be stable over time as shown by Figure 8.

The distribution of hyperbolicity of the quadruplets seems to be relatively independent from the minimum distance in the quadruplet for most of the snapshots studied (Figure 6), while in a grid it linearly depends from that distance. This is however consistent with a tree-like distance.

While observed maximum $\delta$-hyperbolicity is never more than 2 for other graphs, MRINFO data behave very differently: $0.8 \%$ of quaduplets have $\delta=2.5$ and the maximum of the graph is $\delta=5$. This imply the existence of long isometric cycles [5] but we can not explain why.

The conclusion is then that the distances (in hop count) between any four vertices (routers or ASes) in the Internet is, on average, like in a tree, exactly or with and additive error of 1. This allows efficient routing schemes to be used [8]. Krioukov et al. [34], [35] already noticed it was possible in the Internet, thanks to its scale-free structure (degrees and distances distribution), using a modified Thorup-Zwick scheme.

\section{INTERNET MODELS}

We compare our results with various generated graphs, aiming at modeling the AS graphs. They are built with the same number of nodes than the largest bi-connected component of the CAIDA AS graph from January 2009. We used:

- An Erdös-Rényi (ER) random graph with same average degree (6.3) than CAIDA AS graph.

- A random graph whose degree distribution follows a power law with the same exponent as the CAIDA AS graph (it can be generated using [36]).

- A random graph (called "AS Degree Dist") with exactly the same degree distribution than the CAIDA AS graph [36].

- A graph generated using the Barabási-Albert (BA) Preferential Attachment model [29].

Table III shows the value of parameters studied for each model. The lower bounds for the treewidth of these graphs are slightly bigger than the bound of the AS graphs and still comparable to 107, the treewidth of a square grid of the same size. That leads us to the conclusion that the treewidth of the Internet is slightly lower than the treewidth of a random graph of the same size and density. A graph generated to follow exactly the same distribution (by random matching) has a treewidth a bit nearer, but it is not relevant enough. The BA graph has treewidth of the same magnitude as the other random graphs.

Figure 7 gives the observed hyperbolicity for these generated graphs compared with the AS graphs. One can notice that randoms graphs with the same distribution (exactly, or power-law of same exponent) are very close to the AS graph. BA graph is however closer to ER graph.

Our conclusion meets Tangmunarunkit et al. work [17]. From both tree-likeness measurements point of view, the simpler (random) "degree based" generators produce even more accurate simulation than the more sophisticated "structural" generators like Barabási-Albert. Especially, for the hyperbolicity, random graphs are so close to the Internet data than they are a valid model of the Internet with respect to that parameter.

\begin{tabular}{|l|c|c|c|c|c|}
\hline Graph & Avg deg & Max deg & $\beta$ & Hyp. & tw \\
\hline CAIDA AS & 6.31 & 1,815 & 2.19 & 2.0 & $\in[82,473]$ \\
\hline Erdös-Rényi & 6.34 & 18 & - & 2.5 & $\geq 135$ \\
Barabási & 6.00 & 283 & 2.92 & 2.0 & $\geq 130$ \\
AS degree dist. & 6.31 & 1,815 & 2.19 & 1.5 & $\geq 110$ \\
Power Law & 8.97 & 1,507 & 2.19 & 1.5 & $\geq 150$ \\
\hline
\end{tabular}

TABLE III: Statistics for Internet models.

\section{CONCLUSION}

We have observed that AS level snapshots exhibit an important churn over time. This can explain previous observations at the IP level.

We have established that the Internet has a high treewidth both at the AS level and at the router level, i.e, roughly same treewidth as a square grid. This result holds independently of the accuracy of the data available. A better accuracy can only result in a higher treewidth. 

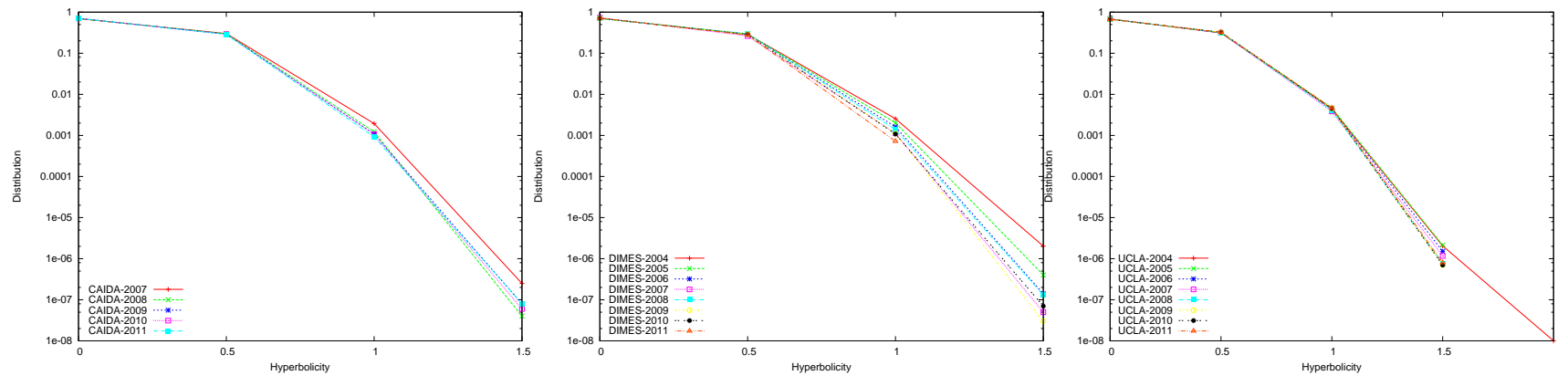

Fig. 8: Variation (or the lack of!) from year to year of hyperbolicity distribution of AS graphs (CAIDA, DIMES and UCLA).

As seen in [4], we observe that the hyperbolicity of almost all Internet snapshots is low. As we use a different hyperbolicity measure, our work comes as a confirmation of this fact. All AS graphs have roughly the same hyperbolicity distribution: for almost all quadruplets it is 0 or 1 . However, we point out that this is not the case for MRINFO data, but we cannot conclude whether it is an artifact of the measurement method. Additionally, we observe that low hyperbolicity is a natural property of power law random graphs which appear again as a simple model capturing many structural properties of the Internet.

\section{REFERENCES}

[1] H. L. Bodlaender, "Dynamic programming on graphs with bounded treewidth," in ICALP, 1988, pp. 105-118.

[2] Y. Shavitt and T. Tankel, "Hyperbolic embedding of internet graph for distance estimation and overlay construction," IEEE/ACM Trans. Netw., vol. 16, no. 1, pp. 25-36, 2008.

[3] V. Ramasubramanian, D. Malkhi, F. Kuhn, M. Balakrishnan, A. Gupta, and A. Akella, "On the treeness of internet latency and bandwidth," in ACM SIGMETRICS, New York, NY, USA, 2009, pp. 61-72.

[4] O. Narayan and I. Saniee, "The large scale curvature of networks," http://arxiv.org/abs/0907.1478v1, 2009.

[5] V. Chepoi, F. F. Dragan, B. Estellon, M. Habib, and Y. Vaxès, "Diameters, centers, and approximating trees of delta-hyperbolicgeodesic spaces and graphs," in Symposium on Computational Geometry, 2008.

[6] V. Chepoi and B. Estellon, "Packing and covering delta -hyperbolic spaces by balls," in APPROX-RANDOM, 2007, pp. 59-73.

[7] C. Gavoille and O. Ly, "Distance labeling in hyperbolic graphs," in ISAAC, 2005.

[8] V. Chepoi, F. F. Dragan, B. Estellon, M. Habib, Y. Vaxès, and Y. Xiang, "Additive spanners and distance and routing labeling schemes for deltahyperbolic graphs," To appear in Algorithmica.

[9] W. Willinger, D. Alderson, and J. C. Doyle, "Mathematics and the internet: A source of enormous confusion and great potential," Notices of the AMS, vol. 56, no. 5, pp. 586-599, 2009.

[10] R. Oliveira, D. Pei, W. Willinger, B. Zhang, and L. Zhang, "The (in)completeness of the observed internet AS-level structure," IEEE/ACM Transac. on Networking, vol. 18, no. 1, pp. 109-122, 2010.

[11] Y. Hyun, B. Huffaker, D. Andersen, E. Aben, M. Luckie, kc claffy, and C. Shanno, "The ipv4 routed /24 as links dataset," 2009.

[12] Y. Shavitt and E. Shir, "Dimes: let the internet measure itself," $S I G$ COMM Comput. Commun. Rev., vol. 35, pp. 71-74, October 2005.

[13] K. Chen, D. Choffnes, R. Potharaju, Y. Chen, F. Bustamante, D. Pei, and Y. Zhao, "Where the sidewalk ends: extending the internet as graph using traceroutes from p2p users," in CoNEXT, 2009, pp. 217-228.

[14] N. Spring, R. Mahajan, D. Wetherall, and T. Anderson, "Measuring isp topologies with rocketfuel," IEEE/ACM Trans. Netw., vol. 12, no. 1, pp. 2-16, 2004.
[15] P. Mérindol, V. V. den Schrieck, B. Donnet, O. Bonaventure, and J.-J. Pansiot, "Quantifying ases multiconnectivity using multicast information," in Internet Measurement Conference, 2009, pp. 370-376.

[16] C. Magnien, F. Ouédraogo, G. Valadon, and M. Latapy, "Fast dynamics in internet topology: preliminary observations and explanations," CoRR, vol. abs/0904.2716, 2009.

[17] H. Tangmunarunkit, R. Govindan, S. Jamin, S. Shenker, and W. Willinger, "Network topology generators: Degree-based vs. structural," in ACM SIGCOMM, 2002, p. 159.

[18] J.-J. Pansiot and D. Grad, "On routes and multicast trees in the internet," Computer Communication Review, vol. 28, no. 1, pp. 41-50, 1998.

[19] R. Govindan and A. Reddy, "An analysis of internet inter-domain topology and route stability," in IEEE INFOCOM, 1997, pp. 850-857.

[20] M. Faloutsos, P. Faloutsos, and C. Faloutsos, "On power-law relationships of the internet topology," in ACM SIGCOMM, 1999, pp. 251-262.

[21] A. Broido and k. claffy, "Internet topology: Connectivity of IP graphs," in Proceedings of SPIE International Symposium on Convergence of IT and Communication, 2001.

[22] Q. Chen, H. Chang, R. Govindan, S. Jamin, S. Shenker, and W. Willinger, "The origin of power-laws in internet topologies revisited," in IEEE INFOCOM, 2002.

[23] N. Robertson and P. D. Seymour, "Graph minors. i. excluding a forest," J. Comb. Theory, Ser. B, vol. 35, no. 1, pp. 39-61, 1983.

[24] T. S. E. Ng and H. Zhang, "Predicting internet network distance with coordinates-based approaches." in IEEE INFOCOM, 2002.

[25] F. Dabek, R. Cox, M. F. Kaashoek, and R. Morris, "Vivaldi: a decentralized network coordinate system." in ACM SIGCOMM, 2004.

[26] M. Gromov, "Hyperbolic groups," in Essays in Group Theory, ser. Math. Sciences Research Inst. Springer, 1987, vol. 8.

[27] B. D. Abrahao and R. D. Kleinberg, "On the internet delay space dimensionality," in IMC, 2008, pp. 157-168.

[28] P. Fraigniaud, E. Lebhar, and L. Viennot, "The inframetric model for the internet," in IEEE INFOCOM, Phoenix, 2008, pp. 1085-1093.

[29] A. L. Barabási and R. Albert, "Emergence of scaling in random networks," Science, vol. 286, no. 5439, 1999.

[30] A. Fabrikant, E. Koutsoupias, and C. H. Papadimitriou, "Heuristically optimized trade-offs: A new paradigm for power laws in the internet," in ICALP, 2002, pp. 110-122.

[31] W. Willinger, R. Oliveira, and B. Zhang, "Quantifying the completeness of the observed internet as-level structure abstract," UCLA, East Lansing, Michigan, Tech. Rep. TR 080026, September 2008.

[32] S. Arnborg, D. G. Corneil, and A. Proskurowski, "Complexity of finding embeddings in a \$ \$-tree," SIAM Journal on Algebraic and Discrete Methods, vol. 8, no. 2, pp. 277-284, 1987.

[33] B. Reed, "Tree width and tangles: A new connectivity measure and some applications," Surveys in combinatorics, vol. 241, pp. 87-162, 1997.

[34] D. V. Krioukov, K. R. Fall, and X. Yang, "Compact routing on internetlike graphs." in IEEE INFOCOM, 2004.

[35] D. V. Krioukov, K. Claffy, K. Fall, and A. Brady, "On compact routing for the internet," ACM SIGCOMM, vol. 37, no. 3, 2007.

[36] F. Viger and M. Latapy, "Efficient and simple generation of random simple connected graphs with prescribed degree sequence," in Computing and Combinatorics, ser. LNCS, 2005, vol. 3595, ch. 45, pp. 440-449. 